\title{
The Performance Analysis of a New Modification of Conjugate Gradient Parameter for Unconstrained Optimization Models
}

\author{
I M Sulaiman ${ }^{1}$, M Mamat ${ }^{1, *}$, M Y Waziri ${ }^{2}$, U A Yakubu ${ }^{3}$, M Malik ${ }^{1}$ \\ ${ }^{1}$ Faculty of Informatics and Computing, Universiti Sultan Zainal Abidin, Terengganu, Malaysia \\ ${ }^{2}$ Department of Mathematics, Faculty of Physical Sciences, Bayero University, Kano, Nigeria \\ ${ }^{3}$ Department of Mathematics, Faculty of Sciences, Maitama Sule University, Kano, Nigeria
}

Received September 13, 2020; Revised October 28, 2020; Accepted November 19, 2020

\begin{abstract}
Cite This Paper in the following Citation Styles
(a): [1] I M Sulaiman, M Mamat, M Y Waziri, U A Yakubu, M Malik, "The Performance Analysis of a New Modification of Conjugate Gradient Parameter for Unconstrained Optimization Models, "Mathematics and Statistics, Vol. 9, No. 1, pp. 16-23, 2021. DOI: 10.13189/ms.2021.090103.
\end{abstract}

(b): I M Sulaiman, M Mamat, M Y Waziri, U A Yakubu, M Malik (2021). The Performance Analysis of a New Modification of Conjugate Gradient Parameter for Unconstrained Optimization Models. Mathematics and Statistics, 9(1), 16-23. DOI: 10.13189/ms.2021.090103.

Copyright $\bigcirc 2021$ by authors, all rights reserved. Authors agree that this article remains permanently open access under the terms of the Creative Commons Attribution License 4.0 International License

\begin{abstract}
Conjugate Gradient (CG) method is the most prominent iterative mathematical technique that can be useful for the optimization of both linear and non-linear systems due to its simplicity, low memory requirement, computational cost, and global convergence properties. However, some of the classical CG methods have some drawbacks which include weak global convergence, poor numerical performance both in terms of number of iterations and the CPU time. To overcome these drawbacks, researchers proposed new variants of the CG parameters with efficient numerical results and nice convergence properties. Some of the variants of the CG method include the scale CG method, hybrid CG method, spectral CG method, three-term CG method, and many more. The hybrid conjugate gradient (CG) algorithm is among the efficient variant in the class of the conjugate gradient methods mentioned above. Some interesting features of the hybrid modifications include inherenting the nice convergence properties and efficient numerical performance of the existing CG methods. In this paper, we proposed a new hybrid CG algorithm that inherits the features of the Rivaie et al. (RMIL*) and Dai (RMIL+) conjugate gradient methods. The proposed algorithm generates a descent direction under the strong Wolfe line search conditions. Preliminary results on some benchmark problems show that the proposed method efficient and
\end{abstract}

promising.

Keywords Conjugate Gradient, Convergence Analysis, Line Search Technique

\section{Introduction}

Given an unconstrained optimization model

$$
\min f(x)
$$

where $f: R^{n} \rightarrow R$ is a smooth function and $g(x) \triangleq \nabla f(x)$ is its gradient, $x \in R^{n}$ is an $n$-dimensional real vector. The CG algorithms are amongst the efficient optimization algorithms for obtaining the solution of problem(1), especially when the dimension $n$ is large [1]. The solution of the unconstrained optimization problem (1) is often in form of a local minimum or global minimal point. In practice, most optimization algorithms achieved only the local minima, because the global minimum is sometimes very difficult to attain as per knowledge of the function is commonly local. Beginning with a starting guess $x_{0} \in R^{n}$, the CG algorithm would generates a sequence of points $\left\{x_{k}\right\}_{k=0}^{\infty}$ using the iterative formula defined as follows

$$
x_{k+1}=x_{k}-\alpha_{k} d_{k}
$$


$\alpha_{k}$ is a step length calculated via suitable line search method along the direction of search $d_{k}$. For the first iteration, the $d_{k}$ is generally the negative of the gradient and known as the direction of steepest descent, i.e. $d_{0}=-g_{0}$. However, resulting $d_{k}$ 's are computed using

$$
d_{k}=-g_{k}+\beta_{k} d_{k-1}
$$

where the scalar $\beta_{k}$ is referred to as conjugate gradient update coefficient [2]. In computing the step size $\alpha_{k}$, some of the inexpensive commonly used line searches algorithms include the weak Wolfe conditions

$$
\begin{gathered}
f\left(x_{k}+\alpha_{k} d_{k}\right) \leq f\left(x_{k}\right)+\delta \alpha_{k} g_{k}^{T} d_{k} \\
g_{k+1}{ }^{T} d_{k} \geq \sigma g_{k}^{T} d_{k}
\end{gathered}
$$

the strong Wolfe (SWP) condition (4) and

$$
\left|g_{k+1}{ }^{T} d_{k}\right| \leq\left|\sigma g_{k}^{T} d_{k}\right|
$$

and the generalized Wolfe line search (4) and

$$
\sigma g_{k}^{T} d_{k} \leq g_{k+1}^{T} d_{k} \leq \sigma_{1} g_{k}^{T} d_{k}
$$

with $0<\delta<\sigma<1$, and $\sigma_{1} \geq 0$ bieng constants that are frequently used. The efficient and effective line search of a CG method gives an approximate value of the step length by guaranteeing that the stages are precisely either too long or too short.

Generally, CG algorithms are characterized by the choice of coefficient $\beta_{k}$. Some of the classical formulas for $\beta_{k}$ are

$\begin{array}{ll}\beta_{k}^{F R}=\frac{g_{k}^{T} g_{k}}{g_{k-1}^{T} g_{k-1}} & \text { Fletcher - Reeves (FR) [3] } \\ \beta_{k}^{P R P}=\frac{g_{k}^{T}\left(g_{k}-g_{k-1}\right)}{g_{k-1}^{T} g_{k-1}} & \text { Polak - Ribiere - Polyak }\end{array}$

(PRP) $[4,5]$

$\beta_{k}^{H S}=\frac{g_{k}^{T}\left(g_{k}-g_{k-1}\right)}{\left(g_{k}-g_{k-1}\right)^{T} d_{k-1}} \quad$ Hestenes - Stiefel (HS) [6]

$\beta_{k}^{L S}=-\frac{g_{k}^{T}\left(g_{k}-g_{k-1}\right)}{d_{k-1}^{T} g_{k-1}}$

Liu -storey (LS) [7]

$\beta_{k}^{C D}=\frac{g_{k}^{T} g_{k}}{d_{k-1}^{T} g_{k-1}}$

Conjugate Descent (CD) [8]

$\beta_{k}^{D Y}=\frac{g_{k}^{T} g_{k}}{\left(g_{k}-g_{k-1}\right)^{T} d_{k-1}}$

Dai - Yuan (DY) [9]

The above formulas can be characterized into two groupings. One group include PRP [4,5], HS [6], and LS [7]. These formulas are regarded as amongst the greatest efficient $\mathrm{CG}$ algorithms for obtaining the solution of large-scale functions. This is as a result of an inbuilt automatic restart feature that helps prevent them from jamming during the computation. Yet, these algorithms may fail to converge to the solution for some problems, and their convergence are until now not established under certain inexact line search conditions. The other group includes FR [3], CD [8], and DY [9] methods. Though, these algorithms possesses strong convergence properties, their numerical performance is often very poor due to the jamming phenomena $[10,11]$.

The above stated drawbacks motivated researchers to study and proposed numerous mofifications of the CG method with the aim of overcoming the lapses encountered by the classical methods. Some of these modifications include the three-term CG algorithms, spectral CG algorithms, the hybrid CG method, and many more. However, most researcher focussed on the hybrid CG algorithms that combine various CG parameters $\beta_{k}$ so as to overcome the weaknesses and exploit the advantages of the parent CG algorithm [12]. Among the earliest hybrid $\mathrm{CG}$ algorithms is $\beta_{k}^{T S}$ developed by Touati-Ahmed and Storey [13] with formula defined as follows

$$
\beta_{k}^{T S}= \begin{cases}\beta_{k}^{P R P} & \text { if } 0 \leq \beta_{k}^{P R P} \leq \beta_{k}^{F R}, \\ \beta_{k}^{F R} & \text { otherwise. }\end{cases}
$$

This method numerically outperformed both methods of PRP and FR in addition to its nice convergence properties. Based on the above discussion, numerous studies and effort have been done focusing on finding new CG methods with not only good efficient numerical perfoamnce, but also, good convergence properties, (see Refs [14 $30,42,44,45,46,48,49,50])$. For the application of the CG method to real-world situation, (see Refs 31-35,43,47). Motivated by the recent trend on conjugate gradient method, this research aim to study a new hybrid CG method that would possess nice convergence features in addition to efficient numerical performance.

The remaining part of the study is planned as follows. In the subsequent section, we derive the proposed CG parameter $\beta_{k}$ using the idea of existing CG algorithm and further present the specific algorithm. Section 3 presents the global convergence analysis with strong Wolfe conditions. Preliminary numerical results are analysed via the performance profiles introduced by Dolan-Morè [36] in Section 4. In conclusion, we presented the summary of the research in Section 5.

\section{A new hybrid CG method and algorithm}

In an attempt to overcome some of the drawbacks discussed above, Rivaie et al. [37] developed a variant of PRP formula by replacing $\left\|g_{k}\right\|^{2}$ in the denominator by $\left\|d_{k}\right\|^{2}$. The coefficient $\beta_{k}$ of RMIL is computed as

$$
\beta_{k}^{R M I L}=\frac{g_{k+1}^{T}\left(g_{k+1}-g_{k}\right)}{\left\|d_{k}\right\|^{2}}
$$

This method possesses an efficient numerical performance because of an in-built restart feature that prevents it from jamming. The authors show that the method converge globally under exact minimization 
condition. Rivaie [38] further defined a vaiant of RMIL method by retain the denominator $\beta_{k}^{R M I L}$ while adding a negative previous $d_{k}$ to the numerator. This method is defined as follows

$$
\beta_{k}^{R M I L *}=\frac{g_{k+1}^{T}\left(g_{k+1}-g_{k}-d_{k-1}\right)}{\left\|d_{k}\right\|^{2}}
$$

This method inherited the nice convergence properties of RMIL algorithm and would reduce to $\beta_{k}^{R M I L}$ under exact minimization conditions. Rivaie et al. [38] further show that the formula is globally convergent provided the SWP condition is satisfied.

Recently, Dai [39] pointed the convergence prove of RMIL [37] is not correct and pointed out that a wrong inequality known to play a vital part in the convergence study of the proposed algorithm. The author further presented as modification of RMIL method as follows:

$$
\begin{aligned}
& \beta_{k}^{R M I L+} \\
& = \begin{cases}\frac{g_{k+1}^{T}\left(g_{k+1}-g_{k}\right)}{\left\|d_{k}\right\|^{2}}, & \text { if } 0 \leq\left|g_{k+1}^{T} g_{k}\right| \leq\left\|g_{k+1}\right\|^{2} \\
0, & \text { otherwise, }\end{cases}
\end{aligned}
$$

Though, the performances of both methods are similar, Dai [38] show that RMIL+ converges globally using exact minimization condition. The convergence RMIL+ was further studied under the strong Wolfe conditions by Yousif [40].

Motivated by the nice convergence properties and efficient numerical performance of [37-40] and taking into account the ideas of the hybrid methods of [13], [21], we suggest a new hybrid CG coefficient as follows:

$$
\beta_{k}^{h R M I L}= \begin{cases}\beta_{k}^{R M I L+} \text { if } 0 \leq \beta_{k}^{R M I L+} \leq \frac{\left\|g_{k+1}\right\|^{2}}{\left\|d_{k}\right\|^{2}}, \\ \frac{g_{k+1}^{T}\left(g_{k+1}-g_{k}-d_{k-1}\right)}{\left\|d_{k}\right\|^{2}} \text { otherwise. }\end{cases}
$$

Next, we give the algorithm of (9) as follows.

\section{Algorithm 1.}

Stage 1. Starting: Assumed $x_{0} \in R^{n}, d_{0}=-g_{0}$, fixe $k:=0$.

Stage 2. Solve for $\alpha_{k}$ using (4) and (6).

Stage 3. Update $x_{k}$ via (2).

Stage 4. Calculate $\beta_{k}$ by (9) and update $d_{k}$ by (3).

Stage 5. If $\left\|g_{k}\right\| \leq 10^{-6}$, terminate. Else, go to stage 2 with $k:=k+1$.

The assumptions given below are very vital in the study and analysis of various CG algorithm convergence properties.

Assumption A. $f(x)$ is bounded from below on the level set

$$
\Omega=\left\{x \in R^{n} / f(x) \leq f\left(x_{0}\right)\right\} .
$$

Assumption B. In some neighborhood $N$ of $\Omega, f$ is smooth and $g(x)$ is Lipchitz continuous in $N$, such that, $\exists L>0$ (constant) satisfying;

$$
\|g(x)-g(y)\| \leq L\|x-y\| \forall x, y \in N .
$$

\section{Global Convergence Analysis}

This part will discuss the convergence of $\boldsymbol{\beta}_{\boldsymbol{k}}^{\boldsymbol{h R M I L}}$. One of the general condition that every CG algorithm should possess is the descent property defined as

$$
g_{k}^{T} d_{k} \leq-C\left\|g_{k}\right\|^{2}, C>0 .
$$

To ease the theoretical proof, we need to simplify $\boldsymbol{\beta}_{\boldsymbol{k}}^{h R M I L}$ as follows

$$
\beta_{k}^{h R M I L}=\left\{\begin{array}{cc}
\beta_{k}^{R M I L+} \text { if } 0 \leq \beta_{k}^{R M I L+} \leq & \frac{\left\|g_{k+1}\right\|^{2}}{\left\|d_{k}\right\|^{2}} \\
\frac{g_{k+1}^{T}\left(g_{k+1}-g_{k}-d_{k-1}\right)}{\left\|d_{k}\right\|^{2}} & \text { otherwise. }
\end{array}\right.
$$

If $\mathbf{0} \leq \boldsymbol{\beta}_{\boldsymbol{k}}^{R M I L+} \leq \frac{\left\|g_{k+1}\right\|^{2}}{\left\|d_{k}\right\|^{2}}$, then, it is obvious from [39] that

$$
\beta_{k}^{h R M I L}<\beta_{k}^{R M I L+} \leq \frac{\left\|g_{k+1}\right\|^{2}}{\left\|d_{k}\right\|^{2}}
$$

Otherwise,

$$
\begin{array}{r}
\beta_{k}^{h R M I L}=\frac{g_{k+1}^{T}\left(g_{k+1}-g_{k}-d_{k-1}\right)}{\left\|d_{k}\right\|^{2}} \\
=\frac{\left\|g_{k+1}\right\|^{2}-g_{k+1}^{T} g_{k}-g_{k+1}^{T} d_{k-1}}{\left\|d_{k}\right\|^{2}}
\end{array}
$$

From [38], it follows that

$$
0 \leq \beta_{k}^{h R M I L} \leq \frac{\left\|g_{k+1}\right\|^{2}}{\left\|d_{k}\right\|^{2}}
$$

Hence, for both cases, we have

$$
0 \leq \beta_{k}^{h R M I L} \leq \frac{\left\|g_{k+1}\right\|^{2}}{\left\|d_{k}\right\|^{2}} .
$$

The convergence prove of the proposed CG formula is built on (12) and the theorems that would be discussed below.

\subsection{Sufficient Descent Condition}

The following theorems would be used to show that $\beta_{k}^{h R M I L}$ possess (11) under inexact line search.

Theorem 1: For any CG algorithm defined by (2) and (3), with $\beta_{k}$ defined by (9), and $\alpha_{k}$ is calculated using the SWP conditions (4) and (6) with $0<\sigma<\frac{1}{4}$. Then,

$$
\frac{\left\|g_{k}\right\|}{\left\|d_{k}\right\|^{2}}<2, \quad \forall k \geq 0 .
$$

Proof: The prove of this theorem follows from Osman [40].

Theorem 2. For any CG algorithm defined by (2) and (3), with $\beta_{k}$ defined by (9), and $\alpha_{k}$ calculated using the SWP conditions (4) and (6) with $0<\sigma<\frac{1}{4}$. Then, 


$$
-1-2 \sigma \leq \frac{g_{k}^{T} d_{k}}{\left\|g_{k}\right\|^{2}} \leq-1+2 \sigma, \quad \forall k \geq 0 .
$$

Hence, the condition (11) holds.

Proof: For $k=0$, it is obvious that $\frac{\left\|g_{0}\right\|^{2}}{\left\|d_{0}\right\|^{2}}=1<2$ which follows from (13). Hence Theorem 1 is true for $k=0$. Next, we need to also show that Theorem 1 is true for $k>0$.

Case 1: If $0 \leq \beta_{k}^{R M I L+} \leq \frac{\left\|g_{k+1}\right\|^{2}}{\left\|d_{k}\right\|^{2}}$, then for $0 \leq$ $\left|g_{k+1}^{T} g_{k}\right| \leq\left\|g_{k+1}\right\|^{2}$ from [39], we have

$$
\beta_{k}^{h R M I L}=\frac{g_{k+1}^{T}\left(g_{k+1}-g_{k}\right)}{\left\|d_{k}\right\|^{2}}
$$

Multiplying both side of (3) by $g_{k}^{T}$ gives

$$
g_{k}^{T} d_{k}=-\left\|g_{k}\right\|^{2}+\beta_{k}^{h R M I L} g_{k}^{T} d_{k-1},
$$

From (12) and SWP condition (6), we have

$$
\begin{array}{r}
-\sigma \beta_{k}^{h R M I L}\left|g_{k-1}^{T} g_{k-1}\right| \leq \beta_{k}^{h R M I L} g_{k}^{T} d_{k-1} \leq \\
\sigma \beta_{k}^{h R M I L}\left|g_{k-1}^{T} g_{k-1}\right|
\end{array}
$$

Considering (15) and (16) and applying Cauchy Schwartz inequality, we get

$$
\begin{array}{r}
\left\|g_{k}\right\|^{2}-\sigma \beta_{k}^{h R M I L}\left\|g_{k-1}\right\|\left\|d_{k-1}\right\| \leq g_{k}^{T} d_{k} \leq-\left\|g_{k}\right\|^{2}+ \\
\sigma \beta_{k}^{h R M I L}\left\|g_{k-1}\right\|\left\|d_{k-1}\right\|
\end{array}
$$

Substituting $\beta_{k}^{\text {hRMIL }}$ in (17) gives

$$
\begin{gathered}
-\left\|g_{k}\right\|^{2}-\sigma \frac{\left\|g_{k}\right\|^{2}}{\left\|d_{k-1}\right\|^{2}}\left\|g_{k-1}\right\|\left\|d_{k-1}\right\| \leq g_{k}^{T} d_{k} \\
\leq-\left\|g_{k}\right\|^{2}+\sigma \frac{\left\|g_{k}\right\|^{2}}{\left\|d_{k-1}\right\|^{2}}\left\|g_{k-1}\right\|\left\|d_{k-1}\right\|
\end{gathered}
$$

Dividing (18) by $\left\|g_{k}\right\|^{2}$

$$
-1-\sigma \frac{\left\|g_{k-1}\right\|}{\left\|d_{k-1}\right\|} \leq \frac{g_{k}^{T} d_{k}}{\left\|g_{k}\right\|^{2}} \leq-1+\sigma \frac{\left\|g_{k-1}\right\|}{\left\|d_{k-1}\right\|}
$$

From (19) and Theorem 1, it follows

$$
-1-2 \sigma \leq \frac{g_{k}^{T} d_{k}}{\left\|g_{k}\right\|^{2}} \leq-1+2 \sigma .
$$

Case 2: Otherwise, that is, when

$$
\beta_{k}^{h R M I L}=\frac{g_{k+1}^{T}\left(g_{k+1}-g_{k}-d_{k-1}\right)}{\left\|d_{k}\right\|^{2}}
$$

Proof: The proof of case 2 follows from Rivaie et al. [38].

It is worthy to note that the theoretical prove alone is not sufficient to guarantee the efficiency or robustness of any conjugate gradient algorithm. Therefore, there would be need to assess the performance on some benchmark test problems.

The mentioned functions often check the performance of the $\mathrm{CG}$ methods in situation such as long narrow valley. Below are examples of some artificial nonlinear unconstrained optimization functions with two variables. These functions include essentially unimodal functions, functions having significant null-space effects, and Functions with a small number of significant local optima

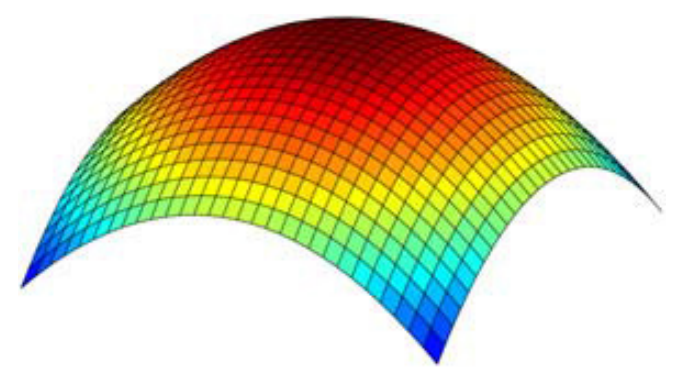

Figure 1. Essentially Unimodal function

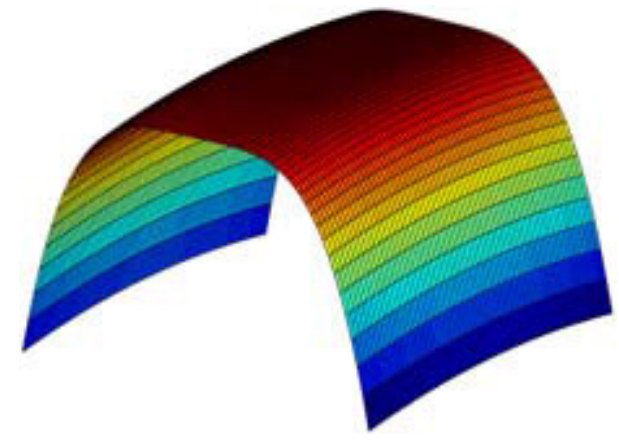

Figure 2. Functions with significant null space

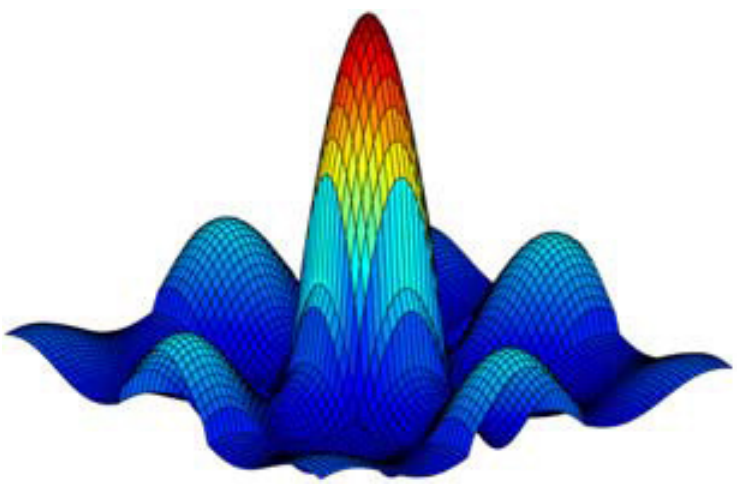

Figure 3. Functions with a small number of significant local optima

These artificial problem functions are very fast to operate, and also their applications help in algorithm development.

\section{Numerical Results}

Here, we studied the performance of defined $h R M I L$ formula by equating with the performance of $R M I L+$ [39] and $R M I L *$ [38] algorithms using 23 benchmark functions taken from Andrei [41]. This comparison was done based on number of iterations and CPU time using the strong Wolfe line search procedures. All problems and formulas are coded and run on the same Matlab programs with termination criterion set as $\left\|g_{k}\right\| \leq 10^{-6}$. All problems have been implement using dimensions ranging from $2 \leq n \leq 10,000$ to illustrate the robustness of hRMIL as shown in Table 1. The performance was also analysed via the performance profile software developed by Dolan and More [36] as can be seen in Figure 6 and 7 . 
Table 1. List of Unconstrained Optimization Test Functions

\begin{tabular}{|c|c|c|c|}
\hline No & Functions & $\mathrm{N}$ & Initial points \\
\hline 1 & Booth & 2 & $(-8,-8)(49,49)(80,80)$ \\
\hline 2 & TRECCANI & 2 & $(-2.1,2)(20,20)(79,79)$ \\
\hline 3 & Zettl & 2 & $(6,6)(20,20)(-100,-100)$ \\
\hline 4 & Raydan 2 & 2,4 & $(1,3)(-17,16)(2,24)$ \\
\hline 5 & Dixon and Price & 2,4 & $(-55,-55)(85,85)(101, \ldots, 101)$ \\
\hline 6 & Hager & 2,4 & $(6, \ldots, 6)(-17, \ldots,-17)(-78, \ldots,-78)$ \\
\hline 7 & $\begin{array}{l}\text { Ext Freudenstein } \\
\text { andRoth }\end{array}$ & $2,4,10$ & $(2, \ldots, 2)(19.2,19.2)(0.5,30)$ \\
\hline 8 & Raydan 1 & $2,4,10$ & $(1, \ldots, 1)(-10, \ldots,-10)(-20, \ldots,-20)$ \\
\hline 9 & Extended penalty & $2,4,10$ & $(2, \ldots, 2)(19, \ldots, 19)(59, \ldots, 59)$ \\
\hline 10 & Extended Maratos & $2,4,10,100$ & $(18, \ldots, 18)(-4.5, \ldots,-4.5)(-84,-106)$ \\
\hline 11 & Generlized Tridiagonal 1 & $2,4,10,100$ & $(1,1)(20,20)(40,40)$ \\
\hline 12 & Extended Beale & $2,4,10,100$ & $(-1.3, \ldots,-1.3)(5, \ldots .5)(11.3, \ldots, 11.3)$ \\
\hline 13 & Extended Denschnb & $2,4,10,100$ & $(3, \ldots, 3)(23, \ldots, 23)(200, \ldots, 200)$ \\
\hline 14 & Extended Tridiagonal 1 & $2,4,10,100,500$ & $(3,3)(8,8)(24.6 \quad 24.7)$ \\
\hline 15 & Generalized Quartic 1 & $2,4,10,100,500$ & $(10, \ldots, 10)(20, \ldots, 20)(80, \ldots, 80)$ \\
\hline 16 & Extended Shallow & $2,4,10,100,500$ & $(11, \ldots, 11)(-1, \ldots,-1)(-50,110)$ \\
\hline 17 & Extended Himmelblau & $2,4,10,100,500$ & $(1,5)(10, \ldots, 10)(41, \ldots, 41)$ \\
\hline 18 & Sum Squares & $2,4,10,100,500$ & $(3.7, \ldots, 3.7)(15, \ldots, 15)(35, \ldots, 35)$ \\
\hline 19 & Qudratic 2 & $2,4,10,100,500,1000$ & $(0.5, \ldots, 0.5)(20, \ldots, 20)(80, \ldots, 80)$ \\
\hline 20 & Diagonal 2 & $2,4,10,100,500,1000$ & $(1, \ldots, 1)(5, \ldots, 5)(15, \ldots, 15)$ \\
\hline 21 & $\begin{array}{c}\text { Extended White and } \\
\text { Holst }\end{array}$ & $2,4,10,100,500,1000$ & $(-1.3, \ldots,-1.3)(10, \ldots, 100)(11, \ldots, 11)$ \\
\hline 22 & Ext Quadratic penalty2 & $2,4,10,100,500,1000$ & $(0.5, \ldots, 0.5)(21, \ldots, 21)(50, \ldots, 50)$ \\
\hline 23 & Extended Rosenbrock & $2,4,10,100,500,1000$ & $(2, \ldots, 2)(20, \ldots, 20)(80, \ldots, 80)$ \\
\hline
\end{tabular}

The set of unconstrained optimization test functions used for numerical computation plays an essential role in the numerical study any CG method. Some of the widely used functions include Treccani function (21) and Raydan 1 function (22) which have in recent been extended to higher dimensions.

$$
\begin{aligned}
& f(x)=\sum_{i=1}^{n} \frac{i}{10}\left(\exp \left(x_{i}\right)-x_{i}\right) \\
& f(x)=\sum_{i=1}^{n} \frac{i}{10}\left(\exp \left(x_{i}\right)-x_{i}\right)
\end{aligned}
$$

The image view of these unimodal functions are given as follows Figure 4 present the Treccani function, while Figure 5 is the Raydan 1 function below.

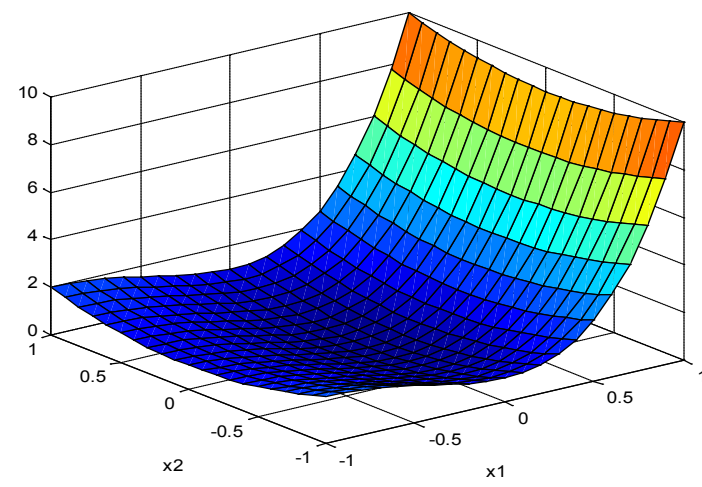

Figure 4. Treccani function

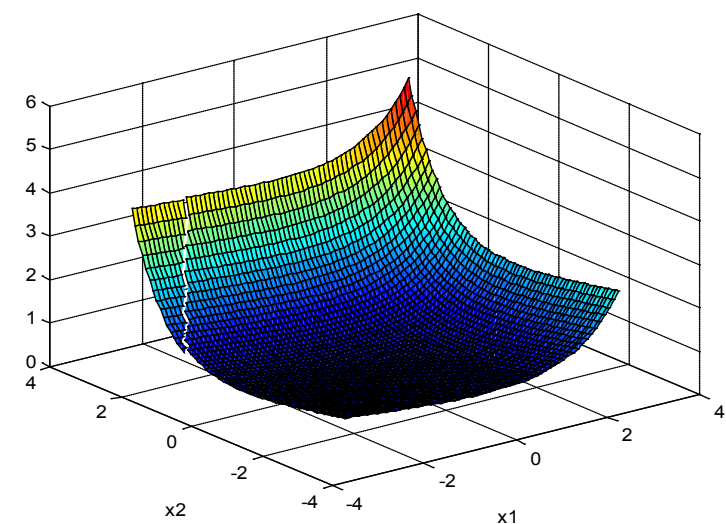

Figure 5. Raydan 1 function

The performance profile is employed to evaluates and compares the performance for the classes of involved solvers $S$ on a whole set of test problems $P$. Assume that $n_{p}$ problems and $n_{s}$ solvers exists, for every solver $s$ and problem $p$, Dolan and More defined

$\tau_{p, s}=$ Computation time (CPU time or NO.IT.) needed by solver $s$ to solve problem $p$.

For each algorithm, the performance software graphs the segment $P$ of each given problem such that the method is in the neighborhood of a factor of $\tau$ of the fastest time. The uppermost curve indicates the algorithm with the best performance. That is, the algorithm that obtained the solutions of nearly all the given function within the shortest time. 


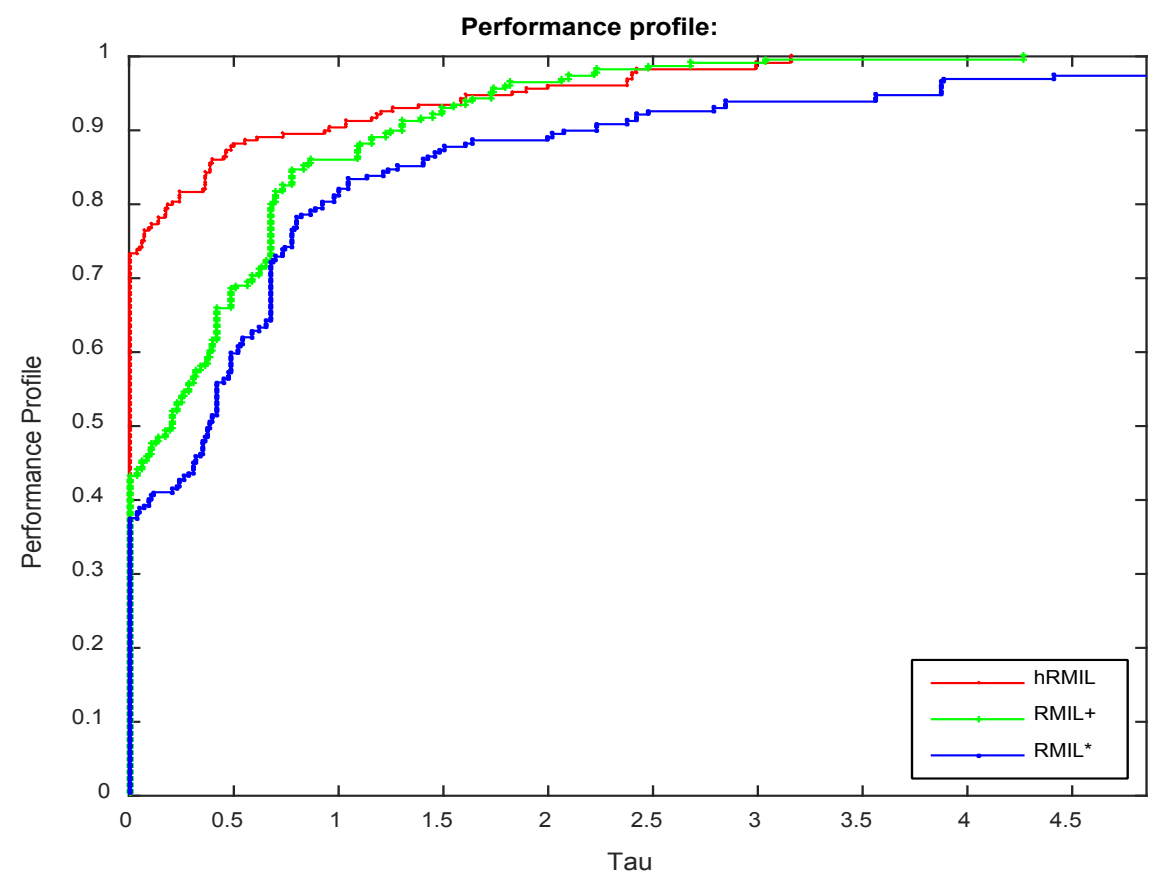

Figure 6. Performance profile with regards to iteration number

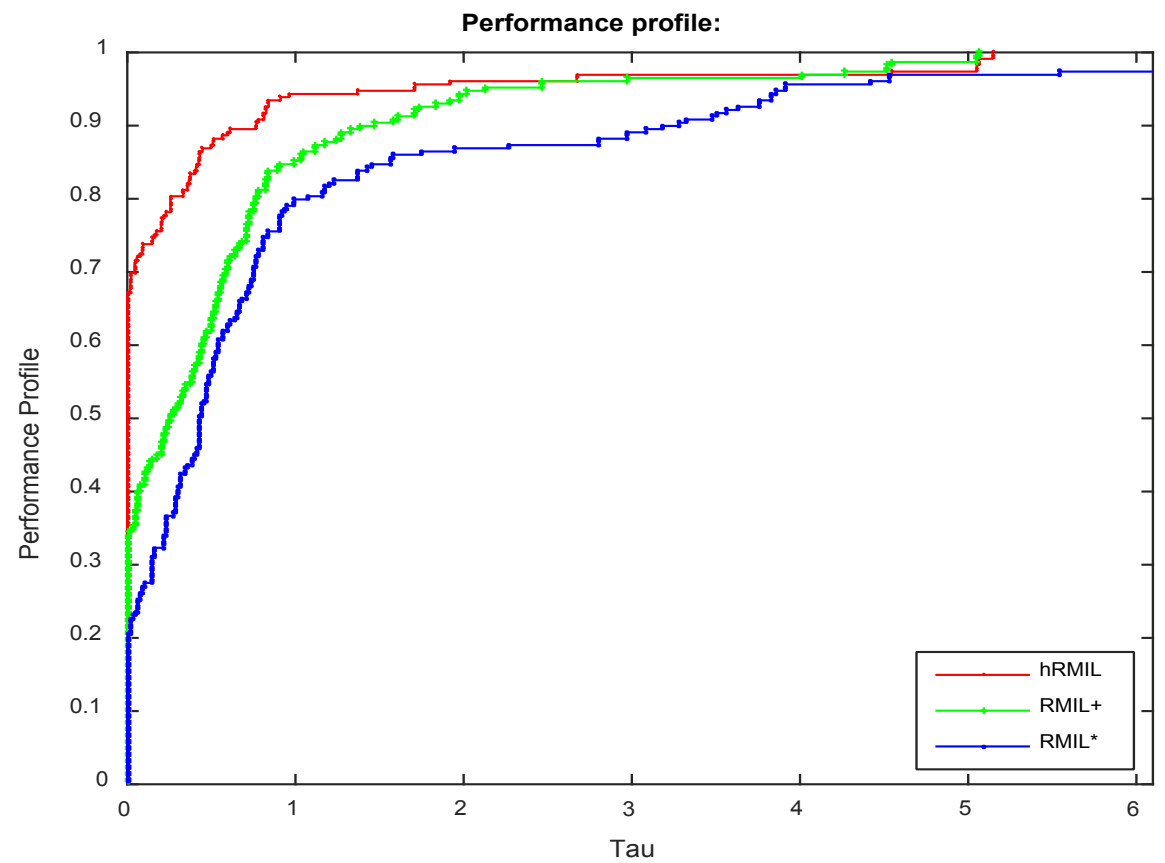

Figure 7. Performance profile with regards to CPU time

Considering both figures, we can see that the derived $h R M I L$ method behaves more like the RMIL+ method. However, $h R M I L$ has the least iterations and CPU time under SWP as can be observe on the left side of both figures. Also, we can notice that hRMIL lies above RMIL+ and RMIL* methods, both under CPU time and iteration number. These show that the hybrid hRMIL algorithm is efficient and promising. Hence, it can be considered as a substitute for solution of optimization models.

\section{Conclusions}

In this paper, based on the nice convergence analysis and efficient numerical performance of previous hybrid CG algorithms, we present an alternative hybrid CG algorithm that inherits the features of the known Rivaie et al. 
(RMIL*), Dai (RMIL+) and Osman (RMIL+) CG algorithms. The convergence analysis of the defined $h R M I L$ was studied under SWP. The performance of $\beta_{k}^{h R M I L}$ was compared with that of the existing $\beta_{k}^{R M I L *}$ and $\beta_{k}^{R M I L+}$ methods on several unconstrained optimization benchmark functions using the performance software by Dolan and More [36]. The computational results show that the proposed $h R M I L$ algorithm is both efficient and promising.

\section{REFERENCES}

[1] Sulaiman, I M., Mamat, M., Waziri, M.Y., Yakubu, A. U., Malik, M. The convergence properties of a new hybrid conjugate gradient parameter for unconstrained optimization models. Journal of Physics Conference Series 1734(012018):1-9, (2021).

[2] Sulaiman, I M., Mamat, M., Kamfa, K., Muktar D. A Descent Modification of Conjugate Gradient Method for Optimization Models. Iraqi Journal of Science. 61(7), 1745-1750, (2020). DOI: 10.24996/ijs.2020.61.7.23.

[3] Fletcher R., Reeves C. Function minimization by conjugate gradients, Comput. J., 7, 149-154, (1964).

[4] Polak. E., Ribiere, G. Note sur la convergence de directions conjug'ees, Rev. Francaise Informat Recherche Opertionelle, 3e ann'ee, 16, 35-43, (1969).

[5] Polyak B. T. The conjugate gradient method in extreme problems, USSR Comp. Math. Math. Phys., 9, pp. 94-112, (1969).

[6] Hestenes, M. R., Stiefel E. L. Methods of conjugate gradients for solving linear systems, J. Research Nat. Bur. Standards, 49, 409-436, (1952).

[7] Liu, Y., Storey, C. Efficient generalized conjugate gradient algorithms, part 1: theory, J.optim.theory Appl. 69, 129-137, (1992).

[8] Fletcher R.. Practical Methods of Optimization, 2nd ed., John Wiley, New York, (1989).

[9] Dai, Y., Yuan, Y. A nonlinear conjugate gradient with strong global convergence properties, SIAM J.optim, 10, 177-182, (2000).

[10] Hager, W. W., Zhang, H. A survey of nonlinear conjugate gradient methods. Pac. J. Optim., 2, 35-58, (2006).

[11] Gilbert J. C and Nocedal J. 1992. Global convergence properties of conjugate gradient methods for optimization, SIAM. J. Optim., vol 2, pp. 21-42.

[12] Kaelo, P., Mtagulwa, P., Thuto, M.V. A globally convergent hybrid conjugate gradient method with strong Wolfe conditions for unconstrained optimization. Math Sci 14, 1$9,(2020)$.

[13] Touati-Ahmed, D., Storey, C. Efficient hybrid conjugate gradient techniques. J. Optim. Theory Appl. 64(2), 379-397 (1990).
[14] Liu, G. H., Han J. Y., Yin H. X. Global convergence of the Fletcher-Reeves algorithm with an inexact line search, Report, Institute of Applied Mathematics, Chinese Academy of Sciences, Beijing, (1993)

[15] Hager, W.W., Zhang, H. A new conjugate gradient method with guaranteed descent and an efficient line search, SIAMJ. Optim., 16, 170-192, (2005).

[16] Aini, N. Rivaie, M. Mamat, M., Sulaiman, I M. A Hybrid of Quasi-Newton Method with CG Method for Unconstrained Optimization. J. Phys.: Conf. Ser. 1366(012079), (2019).

[17] Yu, G. H., L. Guan, L.T., Li, G.Y. Global convergence of modified Polak-Ribière-Polyak conjugate gradient methods with sufficient descent property. J. Ind. Manag. Optim., 4, 565-579, (2008).

[18] Saleh, N.A., Sulaiman, I.M., Mamat, M., Deiby, T.S. Nelson, N. A New Hestenes-Stiefel and Fletcher-Reeves Conjugate Gradient Method with Descent Properties for Optimization Models. International Journal of Supply and Operations Management, 7(4), 344-349, (2020).

[19] Alhawarat, A., Salleh, Z., Mamat, M., Rivaie, M. An efficient modified Polak-Ribiere-Polyak conjugate gradient method with global convergence properties. Optim. Methods Softw. 32(6), 1299-1312 (2017).

[20] Babaie-Kafaki, S. A quadratic hybridization of Polak-Ribie'e're-Polyak and Fletcher-Reeves conjugate gradient methods. J. Optim. Theory Appl. 154(3), 916-932 (2012).

[21] Salih, Y., Hamoda, M.A., Rivaie, M. New hybrid conjugate gradient method with global convergence properties for unconstrained optimization. Malays. J. Comput. Appl. Math. 1(1), 29-38 (2018).

[22] Dai, Y.H., Yuan, Y.X. An efficient hybrid conjugate gradient method for unconstrained optimization. Ann. Oper. Res., 103, 33-47, (2001).

[23] Talat, A., Sulaiman, I.M., Mamat, M., Rivaie, M. An Efficient Hybrid Conjugate Gradient Coefficient under Inexact Line Search. International Journal of Advanced Trends in Computer Science and Engineering, 9(1), 784-788 (2020).

[24] Andrei, N. Hybrid conjugate gradient algorithm for unconstrained optimization. J. Optim. Theory Appl., 141, 249-264, (2009).

[25] Kamfa, K., Waziri, M.Y., Sulaiman, I.M., Ibrahim, M.H., Mamat, M., S.S. Abas. An Efficient Hybrid BFGS-CG Search Direction for Solving Unconstrained Optimization Problems. Journal of Advanced Research in Dynamical and Control Systems 12(2):1035-1041 (2020).

[26] Touati-Ahmed, D., Storey. C. Efficient hybrid conjugate gradient techniques. J. Optim. Theory Appl., 64, 379-397, (1990).

[27] Malik, M., S.S. Abas., Mamat, M., Sukono, Sulaiman, I.M. A New Hybrid Conjugate Gradient Method with Global Convergence Properties. International Journal of Advanced Science and Technology, 29(05), 199-210 (2020).

[28] Xu, X., Kong, F.: New hybrid conjugate gradient methods with the generalized Wolfe line search. SpringerPlus 5(1), 881 (2016). 
[29] Mtagulwa, P., Kaelo, P.: An efficient modified PRP-FR hybrid conjugate gradient method for solving unconstrained optimization problems. Appl. Numer. Math. 145, 111-120 (2019)

[30] Jiang, X.Z., Han, L., Jian, J.B. A globally convergent mixed conjugate gradient method with Wolfe line search. Math. Numer. Sin., 34, 103-112 (2012).

[31] Abdulkarim, H.B., Kumam, p., Auwal, B.A., Jirakitpuwapat, W., Abubakar, J.

A. hybrid conjugate gradient algorithm for constrained monotone equations with application in compressive sensing. Heliyon. 6(3): e03466 (2020).

[32] Sulaiman, I.M., Usman, A.Y., Mamat, M. Application of Spectral Conjugate Gradient Methods for Solving Unconstrained Optimization Problems. An International Journal of Optimization and Control: Theories \& Applications, 10(2), 2146-5703 (2020).

[33] Auwal, B.A., Kumam, p., Aliyu, M. A. Global convergence via descent modified three-term conjugate gradient projection algorithm with applications to signal recovery. Results in Applied Mathematics 4:100069, (2019).

[34] Umar, A.O., Sulaiman, I.M., Mamat, M., Waziri, M.Y. Foziah, H.M., Altien, J.R., Deiby, T.S. New Hybrid Conjugate Gradient Method for Solving Fuzzy Nonlinear Equations. Journal of Advanced Research in Dynamical and Control Systems 12(2):585-590 (2020).

[35] Mamat, M., Rokhayati, Y., Noor, N.M.M., Mohd, I. Optimizing human diet problem with fuzzy price using fuzzy linear programming approach. Pakistan Journal of Nutrition, 10(6), 594-598 (2011).

[36] Dolan, E.D., More, J.J. Benchmarking optimization software with performance profiles, Mathematical Programming, 91, 201-213 (2002).

[37] Rivaie, M., Mamat, M., June, L.W., Ismail, M. A new class of nonlinear conjugate gradient coefficients with global convergence properties. Applied Mathematics and Computation, 218(22), 11323-11332 (2012).

[38] Rivaie, M., Mamat, M., Abashar, A. A new class of nonlinear conjugate gradient coefficients with exact and inexact line search. Applied Mathematics and Computation, 268, 1152-1163 (2015).

[39] Dai, Z. Comments on a new class of nonlinear conjugate gradient coefficients with global convergence properties. Applied Mathematics and Computation, 276, 297-300 (2016).

[40] Osman, O.O.Y. The convergence properties of RMIL + conjugate gradient method under the strong Wolfe line search. Applied Mathematics and Computation, 367, 124777 (2020).

[41] Andrei, N. An unconstrained optimization test functions collection. Adv. Model. Optim, 10(1), 147-161 . (2008).

[42] Sulaiman, I.M., Mamat, M., Owoyemi, A.E., Ghazali, P.L., Rivaie, M., Malik, M. "The convergence properties of some descent conjugate gradient algorithms for optimization models" Journal of Mathematics and Computer Science, 22(3), 204-215, (2020).

[43] Yakubu, U.A., Sulaiman, I.M., Mamat, M., Ghazali, P.L., Khalid, K. The convergence properties of a descent conjugate gradient method. Journal of Advanced Research in Dynamical and Control Systems, 12(2), 1011-1016, (2020).

[44] Abashar, A., Mamat, M., Rivaie, M., Mohd, I. Global convergence properties of a new class of conjugate gradient method for unconstrained optimization. Applied Mathematical Sciences Issue 65-68, 3307-3319, (2014).

[45] Sulaiman, I.M., Mamat, M., Owoyemi, A. E., Olowo, E.S., Zamri, N. A class of spectral conjugate gradient method with descent condition for unconstrained optimization. Journal of Advanced Research in Dynamical and Control Systems, 12(2), 2480-2486, (2020).

[46] Sulaiman, I.M., Supian, S., Sukono, F., and Mamat, M. New class of hybrid conjugate gradient coefficients with guaranteed descent and efficient line search. In IOP Conference Series: Materials Science and Engineering, vol. 621 , no. 1, (2019), art. Id. 012021

[47] Sulaiman, I.M. and Mamat, M. A New Conjugate Gradient Methods with Descent Properties and its Application to Regression analysis. Journal of Numerical Analysis, Industrial and Applied Mathematics. 14(1-2), 25-39, (2020).

[48] M. Malik, S. S. Abas, M. Mamat, Sukono, I.M. Sulaiman. A New Hybrid Conjugate Gradient Method with Global Convergence Properties. International Journal of Advanced Science and Technology. 29(05), 199-210, (2020).

[49] M. Malik, M. Mamat, S. S. Abas, I.M. Sulaiman, Sukono. A New Coefficient of the Conjugate Gradient Method with the Sufficient Descent Condition and Global Convergence Properties. Engineering Letters 28(3):1-11.

[50] K. Ghazali1, J. Sulaiman, Y. Dasril, D. Gabda. An Alternative Approach for Finding Newton's Direction in Solving Large-Scale Unconstrained Optimization for Problems with an Arrowhead Hessian Matrix. Mathematics and Statistics 8(2A): 40-46, (2020). 